ISSN (e)-2347-176x ISSN (p) 2455-0450

crossrefDOI: https://dx.doi.org/10.18535/jmscr/v7i3.35

Original Research Article

\title{
Comparative Study on the effect of Medications in Acne Vulgaris and Assessment of Medication Adherence and Quality of life of Acne Patients in a Multi Specialty Tertiary Care Centre \\ Authors
}

\author{
Bincy Baby $^{1}$, Sadma Vijayakumar ${ }^{2}$, P. L. Pranavya ${ }^{3}$, C. D. Shaji Selvin ${ }^{4}$ \\ ${ }^{1,2,3}$ Sreekrishna College of Pharmacy \& Research Centre, Parassala, Thiruvananthapuram, Kerala, India \\ ${ }^{4}$ St. John's College of Pharmaceutical Sciences and Research, Kattappana South, Idukki, Kerala, India \\ *Corresponding Author \\ C. D. Shaji Selvin \\ Email: shajimpharm@gmail.com
}

\begin{abstract}
Background: Acne vulgaris is the most common inflammatory disorder of pilosebaceous glands and has a substantial impact on patients' quality of life. Systemic antibiotics are known to be effective in its treatment. Adherence to acne medication is poor and is a major reason why treatment plans are ineffective. This study is undertaken to compare the efficacy of oral Azithromycin and Tetracycline's and to assess the quality of life and medication adherence among acne vulgaris patients.

Methods: A prospective observational study was conducted with 160 acne vulgaris patients for a period of six months in the Dermatology Dept. of a multispecialty tertiary care hospital. The Global Acne Grading System, Cardiff Acne Disability Index, ECOB questionnaire was used to study efficacy, quality of life and medication adherence respectively.

Results: It was found that 13-20 years age group, females, students were more affected with acne. Most of the patients have family history of acne, have oily skin and non-vegetarian food habit. Smoking and alcohol habit have a significant impact on acne vulgaris. Regarding with the efficacy, azithromycin has better efficacy than tetracycline. Acne has significant impact on the quality of life of majority of patients' especially in females before treatment and after the treatment it was improved. The medication adherence assessment reveals Azithromycin group has showed a slightly better medication adherence than Tetracycline group.

Conclusion: Results of the present study demonstrated that azithromycin, a long acting macrolide, can be an acceptable alternative for tetracycline in treatment of inflammatory acne and given the long half-life, low complications and higher patient compliance.

Keywords: Acne vulgaris, Azithromycin, Tetracyclines, Medication adherence, Quality of life.
\end{abstract}

\section{Introduction}

Acne vulgaris is one of the dermatological condition, generally experienced by majority of the population particularly in their adolescents' stage $^{(1),(2)}$. It affects both sex, but males are most susceptible comparing with females ${ }^{(3)}$.It is a chronic 
inflammatory condition ${ }^{(4)}$ due to the abnormalities in keratinization, functioning of sebaceous glands ${ }^{(5)}$ and infection of bacteria, Propionibacterium acnes $^{(6)}$. Acne affects mainly the face, obviously, facial appearance is an important aspect of an individual's realization of body image and also severe acne affects the self-confidence and may lead to depression and anxiety, thus have a greater impact on the quality of life of affected ${ }^{(1),(7)}$. Various novel approaches such as blue light phototherapy, retinoic acid metabolism blocking agents and suppressants of Th-1 cytokines receives significant attention in the management of acne $\mathrm{e}^{(5),(6)}$. Importantly, Oral antibiotic therapy is widely in routine. Macrolide antibiotic such as Roxithromycin and Tetracycline derivatives are commonly found in treatment option ${ }^{(8)}$. Thus the present study was designed to compare the efficacy of oral azithromycin with tetracycline derivatives such as minocycline and doxycyclinein acne vulgaris patients and also to assess their quality of life and medication adherence.

\section{Methods}

After getting proper consent from institutional ethical committee (R/ADMIN-08/0), a prospective, observational study was carried out for a period of six months, from January 2017 to June 2017 in the Dept. of Dermatology, Cosmopolitan Hospitals Pvt. Ltd., a 550 bedded multispecialty tertiary care centre located in Thiruvananthapuram, Kerala, south India with the aim to compare the effects of orally given Azithromycin with Tetracyclines in acne management. It was also decided to assess the medication adherence and quality of life of the patients.

Totally 160 patients aged between $13-35$ years, affected with moderate to severe acne vulgaris, have complete medical records and willing to participate in the study were included in the study. Patients not belong to the above specified age group, nonwilling patients, pregnant and lactating women, patients with incomplete medical records and known hypersensitivity to antibiotics and other comorbidities were excluded from the study. The participants were informed about the study and their written consent was collected properly. In this study, various tools such as Global acne grading system (GAGS), Cardiff acne disability index (CADI) and ECOB (Elaboration d'un outild' evaluation de l'observance des traitements medicamenteux) and patient data collection form properly designed with the help of subject experts were used for the data collection. The collected data was analyzed by using SPSS software. Comparison of efficacy ofboth drugs were done using the nonparametric test for paired data, Wilcoxon Mann Whitney U test. ' $t$ ' test was used to assess the quality of life.

\section{Results and Discussion}

A prospective, observational study was conducted for a period of six months from January -2017 to June - 2017 among the 160 patients with acne vulgaris in the Dept. of Dermatology Cosmopolitan Hospitals Pvt. Ltd., Thiruvananthapuram, Kerala. Base line data collected is shown in Table 1. It showed thatthe majority of the study subjects were belongs to the age group of 13-20 years (50.00\%). Among them 57.50\% were treated with azithromycin and $42.50 \%$ with tetracycline. The median age at which acne vulgaris develop is found to be between 21-28yrs (31.25\%), among them equal number of patients were $(31.20 \%)$ treated with azithromycin and tetracyclines. Acne vulgaris was found to least developed (18.75\%) in the age group of 29-35 years. A similar result can found in the studies of ${ }^{(1)}$ and ${ }^{(9)}$. The distribution of gender showed that, about 100 patients $(62.50 \%)$ were females and 60 patients $(37.50 \%)$ were males. Among 100 females $67.50 \%$ were prescribed with azithromycin and $57.50 \%$ with tetracyclines. $32.50 \%$ males were prescribed with azithromycin and $42.50 \%$ with tetracyclines. From our study we 
found that females $(62.50 \%)$ were more affected with acne vulgaris than males (37.50\%). Studies of ${ }^{(10)}$ revealed a similar results. This may be because females are more conscious of their appearance than males. While observing the occupational influence of acne vulgaris, students were more affected with acne vulgaris (53.12\%) compared to other professions such as IT, among them $60.00 \%$ were treated with azithromycin and $46.20 \%$ with tetracyclines. $6.25 \%, 10.62 \%$ and $30.00 \%$ patients were found affected in IT profession, house wife and other professions respectively. Occupation impact on acne was significant in studies done by ${ }^{(9)}$ who reported $3.05 \%$ unemployment, though there was no unemployment reported in our study. The increased incidence of acne in students may be due to age, stress, hormones, emotional status etc. In our study, it was found that there are $145(91 \%)$ non-vegetarians and 15 (9\%) vegetarians. Among non-vegetarians $92.50 \%$ were treated with azithromycin and $88.80 \%$ with tetracyclines. $7.50 \%$ vegetarians were treated with azithromycin and $11.20 \%$ with tetracyclines. Relationship between acne and diet is highly controversial. The studies of ${ }^{(2)}$ reported a positive association between food and acne. The small number of vegetarians in this study may be due to large number of non-vegetarians in our society. In our study $8 \%$ patients were smokers, 5\% were alcoholics and $4 \%$ were both. Among smokers $1.20 \%$ were treated with azithromycin and $13.80 \%$ with tetracyclines. $6 \%$ alcoholics were treated with azithromycin and $4 \%$ with tetracyclines. Majority of the patients were found to be not having any social history at all, among them $91.20 \%$ treated with azithromycin and $78.80 \%$ with tetracyclines. Relationship between smoking and acne is unclear. However, the pathogenesis may be due to the effect of nicotine on nicotinic cholinergic receptors ${ }^{(3)}$. Beer, wine and alcohol in large amount worsening the acne, possibly due to the generally increased inflammatory reactivity of the skin after alcohol.
Out of 160 patients, $62.50 \%$ patients found to have family history of acne vulgaris. Among them 25\% have mother with acne, $12.5 \%$ have father with acne, $11.25 \%$ have siblings with acne, $3.13 \%$ have mother and father with acne, $6.25 \%$ have mother and sibling with acne, $4.37 \%$ have father and sibling with acne. $37.50 \%$ patients were found to have no family history of acne vulgaris. Among them $35.00 \%$ were treated with azithromycin and $43.80 \%$ with tetracyclines. In our study, patient with oily skin $(56 \%)$ were found to be more, among them $61.20 \%$ were treated with azithromycin and $51.20 \%$ with tetracyclines. $31 \%$ patients have normal skin and $13 \%$ have dry skin. Many factors were mentioned by acne patients as aggravating their acne condition. Of the 160 patients with acne, 78 $(48.75 \%)$ claimed that their acne becomes worse by using cosmetics, while $82(51.25 \%)$ noticed no effect. Emotional factors such as stress were mentioned by $68.75 \%$ of acne patients. Also, exposure to sunlight and excessive heat during summer time were believed to aggravate acne in $61.25 \%$ and $75 \%$ of patients respectively. Many acne patients $(59.38 \%)$ claimed that excessive sweating was an exacerbating factor for their acne. Among 60 male acne patient, 40 patients believed that shaving exacerbate their acne. Threading and waxing were mentioned by $35(35 \%)$ and $30(30 \%)$ patients out of 100 female patients respectively. Premenstrual exacerbation of acne was experienced by $90 \%$ of female acne patients, while $10 \%$ of them had among dietary factors, most acne patients believed that their acne was exacerbated by eating fatty food, oily food, milk and dairy products $(56.25 \%)$, chocolates $(84.9 \%)$, hyper-glycemic food $(53.13 \%)$ and spicy food (10.8\%). In addition, $87.5 \%$ of acne patients noticed that their acne condition became worse after using oils for different purposes like for cooking, as moisturizers, revitalizers (as body rubs), for hair dressing etc. Acne is typically vacillating in its course. There are periodic flares, some of which may be accounted 
for by various triggers and aggravating factors. It is therapeutically rewarding to identify the concerned triggers and aggravating factors and be able to deal with them. In India, use of vegetable oils, coconut oil, almond oil, olive oil, and rye oil is popular. Oils form an occlusive film over the applied area may cause acne in the acne-prone areas. Oils also cause folliculitis which sometimes complicates preexistent acne. Facials are popular and are regularly undertaken by women in urban areas for a feeling of freshness, rejuvenation, suppleness of skin, tightening of skin, and for delaying the onset of wrinkles, friction and pressure from helmets and tight collars, follicular irritation from shaving, waxing, threading, and laser hair removal sometimes associated with papules and pustules (Table 2). Acne lesions, comedones, papules, pustules and nodules were distributed all over the face, the upper back and the chest of our patients. These areas are known to be enriched with sebaceous gland that provide a lipid rich environment for proliferation of $P$. acnes bacteria, which contribute to inflammatory process of acne. Acne patients were divided into 3 groups according to severity of their acne condition using the GAGS system and found that $43.10 \%$ patients have grade 4, very severe acne; $38.10 \%$ patients have grade 3 , severe acne and $18.80 \%$ patients have grade 2, moderate acne. The reports of the study by ${ }^{(4)}$ revealed that $52 \%$ have mild acne, $34 \%$ have moderate acne, and $6 \%$ have severe acne. The face was common site of grade 2 and grade 3 acne, while upper back and chest where common sites in the grade 4 acne. Come dones, papules and pustules where distributed over all these areas, but nodules were seen on the back and chest than the face. The significant difference in grade 4 acne with grade 2 and grade 3 in our study may be due to grade 4 acne patients seek medical attention more commonly than other grades because of their highly impaired quality of life. In the present study, there are three samples; one is azithromycin and two samples of tetracycline; - minocycline and doxycycline. 50\% patients were prescribed with azithromycin and $37.50 \%$ patients with minocycline and $12.50 \%$ patients with doxycycline. In the comparison of efficacy, among 80 patients in Azithromycin group it was found that $33(41.25 \%), 37(46.25 \%), 9$ $(11.25 \%)$ and $1(1.25 \%)$ patients exhibited complete, marked, moderate and mild improvement respectively. While Tetracyclines proved 25 (31.25\%), 32 (40\%), $16(20 \%), 6(7.5 \%)$ and 1 $(1.25 \%)$ patients with complete, marked, moderate, mild and insignificant improvement respectively. Hence it can be concluded that the Azithromycin exhibit greater proportion of complete improvement followed by marked improvement than Tetracyclines. The efficacy of antibiotics among acne vulgaris patients who were prescribed with Azithromycin was found to be excellent significantly $(\mathrm{p}<0.05)$. The results are shown in Table 3. Analysis of influence of gender on quality of life revealed that 66 female study subjects and 45 males have large effect and 34 females and 15 males has small effect on the quality of life. Hence it is evident that acne has greater impact of quality of life of females compared to males. The results indicated that before treatment and patient counseling, acne has large effect on quality of life of $70 \%$ patients and small effect on $30 \%$ patients. Among grade 4 acne patients $53.20 \%$ have large effect and $20.40 \%$ have small effect. In grade 3 patients $37.80 \%$ have large effects and $38.80 \%$ patients has small effect. In grade 2 patients $9.00 \%$ have large effects and $40.80 \%$ have small effect. Hence it is evident that greater the grade "severity" of acne, greater the level of impairment of quality of life. However, after treatment and patient counseling it was found that, acne has large effect on quality of life of $20 \%$ patients and small effect on $80 \%$ patients. Among grade 4 acne patients $20 \%$ have large effect and $1.50 \%$ have small effect. In grade 3 patients $33.30 \%$ have large effects and $5.40 \%$ patients have small effect. In grade 2 patients 
$26.70 \%$ have large effects and $27.70 \%$ have small effect. In grade 1 patients $13.30 \%$ have large effect and $46.20 \%$ have small effect. Among grade 0 patients $6.70 \%$ have large effect and $19.20 \%$ have small effect. Hence it is evident that, reduction in the grade or "severity" of acne is associated with reduction in level of impairment of quality of life. The CADI score comparison showed that before treatment and patient counseling $70 \%$ patients' exhibit large effect on quality of life and small effect in $30.00 \%$ patients. After treatment and patient counseling $20.00 \%$ exhibit large effect and small effect in $80.00 \%$ patients. Thereby it suggests that greater proportion of patients exhibited better quality of life after treatment and patient counseling than before treatment and patient counseling. The quality of life among acne vulgaris patients after treatment and patient counseling was found to be improved significantly $(\mathrm{p}<0.05)$ with a standard deviation of 3.368. Adherence is essential for any acne treatment to be effective. However poor adherence with anti-acne treatment is primary problem encountered by dermatologist in the course of managing their acne patients, particularly as a high proportion are teenagers, poorly designed treatment program may be a popular reason of treatment failure in patients with acne. In the present study, comparison of medication adherence shows that $49.50 \%$ and $50.80 \%$ patients who were prescribed with Azithromycin exhibited poor and good adherence respectively. On the other side, $50.50 \%$ and and $49.70 \%$ patients who were prescribed with Tetracyclines exhibited poor and good adherence respectively. Hence it shows that there is small difference in medication adherence between the two groups, that is, azithromycin group having slightly greater medication adherence.

Table 1: Baseline data of study subjects affected with acne vulgaris

\begin{tabular}{|c|c|c|c|c|}
\hline \multirow[b]{2}{*}{ Age (In years) } & \multirow[b]{2}{*}{ Count } & \multirow[b]{2}{*}{ Percentage } & \multicolumn{2}{|c|}{ Drug } \\
\hline & & & Azithromycin (\%) & Tetracycline (\%) \\
\hline $13-20$ & 80 & 50 & 57.50 & 42.50 \\
\hline $21-28$ & 50 & 31 & 31.20 & 31.20 \\
\hline $29-35$ & 30 & 19 & 11.20 & 26.20 \\
\hline \multicolumn{5}{|l|}{ Gender } \\
\hline Female & 100 & 62.50 & 67.50 & 57.50 \\
\hline Male & 60 & 37.50 & 32.59 & 42.50 \\
\hline \multicolumn{5}{|l|}{ Occupation } \\
\hline Student & 85 & 53.12 & 60 & 46.20 \\
\hline IT professional & 10 & 6.25 & 6.20 & 6.20 \\
\hline House wife & 17 & 10.62 & 10 & 11.20 \\
\hline Others & 48 & 30 & 23.80 & 36.20 \\
\hline \multicolumn{5}{|l|}{ Diet } \\
\hline Non vegetarian & 145 & 91 & 92.50 & 88.80 \\
\hline Vegetarian & 15 & 9 & 7.50 & 11.20 \\
\hline \multicolumn{5}{|l|}{ Habits } \\
\hline Smoking & 12 & 8 & 1.20 & 13.80 \\
\hline Alcohol & 8 & 5 & 6 & 4 \\
\hline Smoking and alcohol & 4 & 4 & 1 & 4 \\
\hline Nil & 136 & 85 & 91.20 & 78.80 \\
\hline \multicolumn{5}{|l|}{ Family history } \\
\hline Mother & 40 & 25 & 21.20 & 25 \\
\hline Father & 29 & 12.5 & 17.50 & 7.50 \\
\hline Siblings & 18 & 11.25 & 12.50 & 10 \\
\hline Mother \& Father & 5 & 3.13 & 3.80 & 2.50 \\
\hline Mother \& Sibling & 10 & 6.25 & 7.50 & 6.20 \\
\hline Father \& Sibling & 7 & 4.37 & 2.50 & 5 \\
\hline Nil & 100 & 37.50 & 35 & 43.80 \\
\hline \multicolumn{5}{|l|}{ Skin type } \\
\hline Normal & 49 & 31 & 28.80 & 32.50 \\
\hline Dry & 21 & 13 & 10.00 & 16.20 \\
\hline Oily & 90 & 56 & 61.20 & 51.20 \\
\hline
\end{tabular}


Table 2: Effect of aggravating factors in acne vulgaris among study subjects

\begin{tabular}{|c|c|c|c|}
\hline \multirow{2}{*}{\multicolumn{2}{|c|}{ Aggravating factors }} & \multicolumn{2}{|c|}{ Effect on acne vulgaris (\%) } \\
\hline & & Worse & Nil \\
\hline \multicolumn{2}{|l|}{ Oils } & 87.5 & 12.5 \\
\hline \multicolumn{2}{|c|}{ Menstruation $(\mathrm{n}=100)$} & 90 & 10 \\
\hline \multicolumn{2}{|c|}{ Hot weather } & 75 & 25 \\
\hline \multicolumn{2}{|l|}{ Facial massage } & 31.25 & 68.75 \\
\hline \multicolumn{2}{|l|}{ Stress } & 68.75 & 31.25 \\
\hline \multicolumn{2}{|l|}{ Cosmetics } & 48.75 & 51.25 \\
\hline \multicolumn{2}{|l|}{ Sunlight } & 61.25 & 38.75 \\
\hline \multicolumn{2}{|l|}{ Sweating } & 59.38 & 40.62 \\
\hline \multirow{3}{*}{$\begin{array}{l}\text { Friction } \\
\text { (or)pressure:- }\end{array}$} & Shaving $(n=60)$ & 66.66 & 33.33 \\
\hline & Waxing $(n=100)$ & 30 & 70 \\
\hline & Threading $(n=100)$ & 35 & 65 \\
\hline \multirow{4}{*}{ Diet } & Milk and Dairy products & 56.25 & 43.75 \\
\hline & Chocolates & 84.9 & 15.1 \\
\hline & Hyperglycemic foods & 53.13 & 46.87 \\
\hline & Spicy foods & 10.8 & 89.2 \\
\hline
\end{tabular}

Table 3: Comparison of drug efficacy on treatment among study subjects

\begin{tabular}{|l|c|c|c|c|}
\hline \multirow{2}{*}{ Efficacy } & \multicolumn{4}{|c|}{ Drug } \\
\cline { 2 - 5 } & \multicolumn{2}{|c|}{ Azithromycin } & \multicolumn{2}{c|}{ Tetracycline } \\
\cline { 2 - 5 } & Count & Percentage & Count & percentage \\
\hline Complete improvement & 33 & 41.25 & 25 & 31.25 \\
\hline Marked improvement & 37 & 46.25 & 32 & 40 \\
\hline Moderate improvement & 9 & 11.25 & 16 & 20 \\
\hline Mild improvement & 1 & 1.25 & 6 & 7.5 \\
\hline Insignificant improvement & 0 & 0 & 1 & 1.25 \\
\hline
\end{tabular}

\section{Conclusion}

In the present study, efficacy of Azithromycin and Tetracyclines in the treatment, and the quality of life and medication adherence in the treatment of acne vulgaris patients were analyzed. From this study, it was able to found the most susceptible age group and gender, who are at high risk of developing acne and the association of acne with occupation, genetic factors, diet, social history and different aggravating or precipitating factors. The comparison of efficacy shows azithromycin has better efficacy than tetracycline in acne treatment. The study on quality of life reveals that the acne negatively affect it. Females were more affected and greater the grade or "severity" of acne, greater the level of impairment of quality of life. After treatment and patient counselling, a significant improvement was observed. Medication adherence assessment reveals that Azithromycin therapy showed a slightly greater adherence comparing with Tetracyclines treatment. From this results, it was clear that Azithromycin, a long acting macrolide, can be an acceptable alternative for tetracycline in treatment of inflammatory acne and given the long half-life, low complications, and higher patient compliance.

\section{Acknowledgement}

We would like to thank Mr. J. Kumaran, M. Pharm., (Pharmaceutical Biotechnology), for his assistance in the preparation of this manuscript.

\section{Competing Interests: None \\ Funding: None}

\section{References}

1. Arshad Hanisah, Khairani Omar, Shamsul Azhar Shah. Prevalence of acne and its impact on the quality of life in school-aged adolescents in Malaysia. JPrim Health Care. 2009; 1(1): 20-25.

2. Noor Hasnani Ismail, Zahara Abdul Manaf, Noor Zalmy Azizan. High glycemic load diet, milk and ice cream consumption are related to acne vulgaris in Malaysian young adults: a case control study. BMC Dermatol. 
2012; 12:13. Doi: 10.1186/1471-5945-1213.

3. Itay Klaz, Ilan Kochba, Tzipora Shohat, Salman Zarka, Sarah Brenner. Severe acne vulgaris and tobacco smoking in young men. J Investig Dermatol 2006;126(8):1749-1752.

4. Pragya Ashok Nair, Ashok Raman Nair. Quality of life perspective towards acne among adolescents at tertiary care center of Gujarat, India. J Clin Diagn Res. 2015; 9(10): WC01-WC04.Doi: 10.7860/JCDR/2015/14709.6683.

5. Peter C. M. Van De Kerkhof, Marloes M. Kleinpenning, Elke M. G. J. De Jong, Marie-Jeanne P. Gerritsen, Rens J. Van Dooren-Greebe, Hans A. C. Alkemade. Current and future treatment options for acne.J Dermatolog Treat.2006; 17(4): 198204. Doi: 10.1080/09546630600830596.

6. Sadia Ammad, Maria Gonzales, Chris Edwards, Andrew Y Finlay, Caroline Mills. An assessment of the efficacy of blue light phototherapy in the treatment of acne vulgaris. J Cosmet Dermatol. 2008; 7(3): 180-188. Doi: 10.1111/j.14732165.2008.00386.x

7. Sebnem Aktan, Erol Ozmen, Berna S. Anxiety, depression, and nature of acne vulgaris in adolescents. Int $\mathrm{J}$ Dermatol. 2000; 39(5): 354-357

8. Miwa Kobayashi, Kenji Kabashima, Motonobu Nakamura, YoshikiTokura. Effects of oral antibiotic Roxithromycin on quality of life in acne patients. The Journal of Dermatology. 2009; 36(7): 383-391.

9. Rapp SR, Feldman SR, Graham G, Fleischer $\mathrm{AB}$, Brenes G, Dailey M. The acne quality of life index (Acne-QOLI): Development and validation of a brief instrument. Am J Clin Dermatol. 2006; 7(3):185-192.
10. Rapp DA, Brenes GA, Feldman SR, Fleischer ABJr, Graham GF, Dailey M, Rapp SR. Anger and acne: Implications for quality of life, patient satisfaction and clinical care. $\mathrm{Br}$ J Dermatol. 2004; 151(1):183-189. 